\title{
SYMPLECTIC STRUCTURE ON RULED SURFACES AND A GENERALIZED ADJUNCTION FORMULA
}

\author{
T. J. LI AND A. LIU
}

\section{Preliminary}

In this section, we state the theorems needed in our paper. Recently, Seiberg and Witten ([SW], [Wi]) have introduced a new set of 4-manifold invariants. These invariants are in similar spirit to Donaldson invariants but much easier to handle. Various longstanding conjectures including the Thom conjecture are proved using Seiberg-Witten invariants. An important ingredient in the proof of the Thom conjecture by Kronheimer and Mrowka is the wall crossing formula for manifolds with $b_{1}=0$.

Seiberg-Witten invariants take on a very simple form for Kahler surfaces ([Wi], [B], [FM1]). All the basic classes are explicitly known and in particular, the anticanonical bundle is always a basic class. A large part of this story is generalized to symplectic manifolds by Taubes who ([T1], [T2], [T3], [T4]) proved several remarkable theorems on Seiberg-Witten invariants of symplectic four-manifolds. Recall that every symplectic manifold has a complex line bundle, $K$ (called the canonical bundle), which is canonical up to isomorphism. The first theorem of Taubes is

Theorem 1. ([T1]) Let $M$ be an oriented symplectic four-manifold with $b_{2}^{+} \geq 2$. Let $\omega$ be a symplectic form compatible with the orientation. Then $c_{1}\left(K^{-1}\right)$ on $M$ has Seiberg-Witten invariant \pm 1 .

This result clearly shows that the Seiberg-Witten invariant is an important tool to study the differential topology of symplectic four-manifolds; we will see in this paper that indeed this has many applications. The next two theorems of Taubes give very strong constraints on symplectic forms and almost complex structures supporting symplectic structures.

Theorem 2. ([T2]) Let $M, \omega, K$ be as in the above theorem and let $E \in$ $H^{2}(M ; \mathbf{Z})$ have nonzero Seiberg-Witten invariant. Then

$$
|E \cdot[\omega]| \leq c_{1}(K) \cdot[\omega],
$$

Received June 26, 1995. 
and if equality holds then either $\pm E$ is equal to $c_{1}(K)$. In particular,

$$
c_{1}(K) \cdot[\omega] \geq 0
$$

Theorem 3. ([T2]) The manifold $C P^{2}$ has no symplectic form $\omega$ for which $c_{1}(K) \cdot[\omega]>0$.

In his latest paper, for a cohomology class $V \in H^{2}(M ; Z)$, Taubes uses a new kind of Gromov invariant $G r(V)$ (defined by Ruan [R], see also $[\mathrm{MS}]$ and $[\mathrm{RT}]$ ) counting embedded symplectic surfaces (not necessarily connected) in the homology class Poincare dual to $V$.

Theorem 4. ([T3]) $M$ as in Theorem 1. Let $E$ be a nontrivial complex

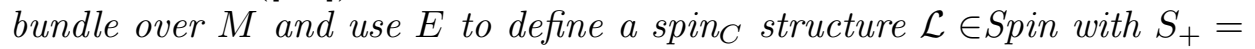
$E \oplus K^{-1} \otimes E$. Then $S W(\mathcal{L})= \pm G r\left(c_{1}(E)\right)$.

Theorem 1 and Theorem 4 are still true when $b_{2}^{+}=1$, if we replace the standard Seiberg-Witten equation by Taubes's $r \rightarrow \infty$ deformed SeibergWitten equation. These theorems make it possible to study the structure of the space of symplectic forms on a symplectic four-manifold.

In complex geometry, the corresponding problem of understanding the moduli space of complex structures has been studied for a long time and there are very satisfactory answers for many complex surfaces([FM2]). For $C P^{2}$, Yau $[\mathrm{Y}]$ proved, as a consequence of his solution of Calabi's conjecture, that any complex surface homotopic to $C P^{2}$ is holomorphically isomorphic to $C P^{2}$.

The moduli space of symplectic structure is a very hard question. Even for $C P^{2}$, not much was known until the middle 80's, when Gromov invented the concept of $J$-holomorphic curves (see [G]) and applied it to prove that if a symplectic homology $C P^{2}$ has a pseudo-holomorphic rational curve which represents the generator of $H^{2}$, then it is symplectomorphic to $C P^{2}$ with the standard symplectic structure.

Gromov's theorem was generalized by Mcduff [M] to cover the case of rational or ruled surfaces assuming the existence of embedded pseudoholomorphic rational curves with nonnegative self-intersection. Although this type of theorem strongly constrains the symplectic structures, at that time, it was not clear at all if the assumption of the existence of the pseudoholomorphic rational curves could be achieved or not.

A striking consequence of Taubes's theorems is that for any possible symplectic structure on $C P^{2}$, the desired pseudo-holomorphic rational curve always exists. Combining with Gromov's result, he proved 
Theorem 5. ([T3]) The manifold $C P^{2}$ has a unique (up to symplectomorphism) symplectic structure.

Another consequence is

Theorem 6. ([T3]) Let $M, \omega, K$ be as in Theorem 1. If $E \in H^{2}(M ; Z)$ $i s$ represented by a smooth -1 sphere, then it is also represented by a symplectic - 1 curve.

\section{Statements of results}

In this paper, we are going to study the symplectic topology of symplectic four manifolds. An important tool is the general wall crossing formula of Seiberg-Witten invariants. If $b_{2}^{+}>1$, then the SW invariants are well defined smooth invariants. However, when $b_{2}^{+}=1$, as in the case of Donaldson invariants, a general one parameter deformation can not avoid the bad points where reducible solutions occur, so the invariance breaks down. It is Kronheimer and Mrowka [KM] who first studied the wall crossing formula when $b_{1}=0$ and the moduli space is zero-dimensional in their proof of the Thom conjecture. In [LL], we generalize their wall crossing formula to allow arbitrary $b_{1}$ and arbitrary dimension of the moduli space as long as it is nonnegative. The difficulties arise because the reducible solutions are not anymore isolated points as in the case $b_{1}=0$. In fact, they are parametrized by the Albanese torus, and the geometry around them is very complicated. By several reductions, we bypass these difficulties and prove

Theorem (General wall crossing formula). Let $M$ be an oriented fourmanifold with $b_{2}^{+}=1$ and $b_{1}$ even, and $\mathcal{L} \in H^{2}(M ; \mathbf{Z})$ a spin $\sin _{C}$ structure with $\operatorname{dim} \mathcal{M}(\mathcal{L}) \geq 0$, then after crossing a wall, $S W(\mathcal{L})$ changes by

$$
\pm\left(\frac{1}{2}\left(\Omega^{2} \cdot \mathcal{L}\right)[M]\right)^{b_{1} / 2} /\left(b_{1} / 2\right) !\left[T^{b_{1}}\right] .
$$

As a corollary, we get

Corollary 1 (Wall crossing formula for ruled surfaces). Let $M$ be an $S^{2}$ bundle over a riemann surface $\Sigma$ of genus $g$ or its blow-up, and let $E \in H^{2}(M ; \mathbf{Z})$ have a Seiberg-Witten moduli space with nonnegative formal dimension $\left(-c_{1}(K) \cdot E+E \cdot E \geq 0\right)$. Then after crossing a wall, $S W(E)$ changes by

$$
\pm\left(\frac{c_{1}(M)+2 E}{2}\left[S^{2}\right]\right)^{g} .
$$

For the precise meaning of $\Omega$, see [LL]. The main conclusion is that when crossing a wall, the invariant does not always change by \pm 1 as in the case 
discussed by Kronheimer and Mrowka, but depends on some characteristic classes calculation. The new phenomenon is the jump of the SW invariant of a line bundle; it only depends on its first Chern class and can change by either an even integer or an odd integer which we call even type and odd type respectively. For an even type line bundle, the SW invariant is still a smooth invariant modulo two.

For a symplectic manifold with $b_{2}^{+}=1$, combined with Taubes's Theorems 1-6, we can often get interesting results without detailed understanding of the geometry of the manifold. This is different from the study of Kahler manifolds which crucially depends on the classification and explicit knowledge of the underlying manifolds. This is illustrated by the following theorem.

Theorem A ( -1 curve theorem). Let $M$ be a symplectic four-manifold with $b_{2}^{+}=1$. If a cohomology class $E$ can be represented by $a-1$ sphere and its paring with $K$ is \pm 1 , then $E$ or $-E$ can be represented by a symplectic sphere.

The proof is inspired by Taubes's proof of Theorem 6. One main application of the explicit wall crossing formula is to study the uniqueness of the symplectic structure on ruled surfaces.

Theorem B. For an $S^{2}$-bundle over a Riemann surface, the symplectic structure is unique (up to diffeomorphism and symplectic deformation).

Theorem C. For blow-ups of geometrically ruled surfaces, the symplectic structures are unique.

Theorem D. There is a unique symplectic structure on $C P^{2} \# k \bar{C} P^{2}$ for $2 \leq k \leq 9$ up to diffeomorphisms and deformation. For $k \geq 10$, The symplectic structure is still unique for the standard canonical class.

We can also show that for geometrically ruled surfaces, the symplectic cone is the union of the ample cones for all different complex structures. Other interesting applications are the following two theorems.

Theorem E (Generalized adjunction inequality). Suppose $M$ is a symplectic four-manifold with $b_{2}^{+}=1$ and $\omega$ is a symplectic form. Let $C$ be a smooth, connected, embedded surface with nonnegative self-intersection. If $[C] \cdot \omega>0$, then $2 g(C)-2 \geq K \cdot[C]+[C]^{2}$.

Theorem F. For a symplectic four-manifold with $b_{2}^{+}=1, \omega_{1}$ and $\omega_{2}$ are two symplectic forms, and $\omega_{1}$ is in the forward cone, $K_{1}$ and $K_{2}$ are canonical classes for $\omega_{1}$ and $\omega_{2}$, if $\omega_{1} \cdot \omega_{2}>0$, then $K_{1} \cdot \omega_{1} \geq K_{2} \cdot \omega_{1}$ where 
equality holds if and only if $K_{1}=K_{2}$ modulo torsion. If $\omega_{1} \cdot \omega_{2}<0$, then $K_{1} \cdot \omega_{1} \geq-K_{2} \cdot \omega_{1}$.

However as the forward cone and backward cone are disjoint, Theorem E does not imply $K_{1} \cdot \omega_{1} \geq\left|K_{2} \cdot \omega_{1}\right|$ which is impossible since it would contradict Taubes's Theorem 3 .

The proof of Theorem E basically follows Kronheimer and Mrowka's proof for $C P^{2}$, but also use Taubes's theorems. Also note that the Thom conjecture for Kahler surfaces was proved by Morgan, Szabo and Taubes $([\mathrm{MST}])$.

This paper is organized as follows. In $\S 2$, we first introduce some definitions. Then we study in detail the wall crossing behaviour in Taubes's perturbation and prove Theorem A. In $\S 3$, we prove Theorems B, C and D. In $\S 4$, we prove Theorems $\mathrm{E}$ and $\mathrm{F}$.

\section{SW invariants for $b_{2}^{+}=1$ and wall crossing}

Let us first give some definitions. Denote by $p$ the quotient map from the space of closed 2-forms to $H^{2}(M ; \mathbf{R})$.

Let $J$ be an almost complex structure on $M$. We say a nondegenerate 2 -form $\omega$ is $J$-compatible if $J$ is $\omega$-compatible, i.e., $\omega(V, J V)>0$ for all nonzero tangent vector $V$. We call $J$ a symplectic almost complex structure if there exists $J$-compatible symplectic forms. For such a $J$, define the symplectic cone $\mathcal{C}_{J}$ of $J$ to be the image of all the $J$-symplectic forms under the map $p$.

Let $K \in H^{2}(M ; \mathbf{Z})$ be the canonical class of a homotopy class of almost complex structures. We say $K$ is a symplectic canonical class if there exists a symplectic almost complex structure in this homotopy class. For a symplectic canonical class $K$, define the symplectic cone $\mathcal{C}_{K}$ to be the union of the symplectic cones $\mathcal{C}_{J}$ of all the symplectic almost complex structures in this homotopy class.

We call two symplectic forms $\omega_{1}$ and $\omega_{2}$ deformation equivalent if $\omega_{1}$ and $\omega_{2}$ are joined by a path of symplectic forms (not necessarily cohomologous). We say that $\omega_{1}$ and $\omega_{2}$ give the same symplectic structure if there exists a self-diffeomorphism $\Phi$ such that $\omega_{1}$ and $\Phi^{*} \omega_{2}$ are deformation equivalent.

For a four-manifold $M$ with $b_{2}^{+}=1$, in general, the Seiberg-Witten invariant is no longer a smooth invariant of the underlying manifold, since a generic 1-parameter deformation can not avoid reducible solutions, i.e., solutions with the spinor part $\psi$ are identically zero. The moduli space has singularities at reducible solutions, and the invariant may jump. Given a metric $g$, since the self-dual harmonic 2-form is one-dimensional, there is a unique associated self-dual harmonic 2 -form $\omega_{g}$ for $g$, $\bmod$ nonzero 
scalars. For the standard SW equation, reducible solutions exist if and only if $P_{+} F_{A}=0$, or equivalently, $c_{1}(L) \cdot\left[\omega_{g}\right]=0$. The cohomology classes with positive square form a cone with two connected components. Given an orientation of $\mathrm{H}^{+}$, one is called the forward cone and the other is called the backward cone.

In the rest of this section, we assume that $M$ is a four-manifold with $b_{2}^{+}=1$. Let us examine in detail Taubes's constructions. There are three parts:

Standard SW equation:

$$
\begin{aligned}
D_{A} \psi & =0 \\
P_{+} F_{A} & =\frac{1}{4} \tau\left(\psi \otimes \psi^{*}\right) .
\end{aligned}
$$

Perturbed SW equation:

$$
\begin{aligned}
D_{A} \psi & =0 \\
P_{+} F_{A} & =\frac{1}{4} \tau\left(\psi \otimes \psi^{*}\right)+t P_{+} F_{A_{0}}-\frac{i t}{4} \cdot \omega \quad 0 \leq t \leq 1
\end{aligned}
$$

where $A_{0}$ is a canonical connection on $K^{-1}$ (up to gauge equivalence).

Deformed SW equation:

$$
\begin{aligned}
D_{A} \psi & =0 \\
P_{+} F_{A} & =\frac{1}{4} \tau\left(\psi \otimes \psi^{*}\right)+P_{+} F_{A_{0}}-\frac{i r}{4} \cdot \omega \quad r \geq 1 .
\end{aligned}
$$

A wall could appear in any of the following three steps,

(1) The standard metric wall: where anti-self-dual harmonic 2-forms suddenly appear.

(2) From the standard SW equation to the perturbed SW equation: there might be some walls for some $t \in[0,1]$.

(3) From the perturbed SW equation to the deformed SW equation: there might be some walls for $r \geq 1$.

In the following, we will study the number of walls that occurred in each step of Taubes' perturbation. We say the wall crossing number of $\mathcal{L}$ is $n$ if after crossing a wall, the SW invariant of $\mathcal{L}$ changes by $\pm n$.

Lemma 2.1. If $c_{1}(E) \cdot \omega \leq 0$ (in particular, if $c_{1}(E) \cdot \omega=0$ ), then there are no walls in step 3.

Proof. Suppose $(A, 0)$ occurs as a reducible solution. Wedge $\omega$ with the deformed equation,

$$
P_{+} F_{A}=P_{+} F_{A_{0}}-i r \omega, \quad r \geq 1
$$


and note that $F_{A}$ is the curvature of $K^{-1} \otimes E^{2}$ and $F_{A_{0}}$ is the curvature of $K^{-1}$. We get

$$
c_{1}\left(K^{-1} \otimes E^{2}\right) \cdot \omega=c_{1}\left(K^{-1}\right) \cdot \omega+r \omega \cdot \omega .
$$

By assumption, $c_{1}(E) \cdot \omega \leq 0$, so $r \leq 0$ and does not lie in the defining region $r \geq 1$.

The above equation, viewed as an equation of $r$, always has a real solution; let $r_{c r}$ be the unique solution. Then we have the following lemma:

Lemma 2.2. Suppose some walls occur in step 2, then

$$
(1-t) c_{1}\left(K^{-1} \otimes E^{2}\right) \cdot \omega=\left(1-r_{c r}\right) t \omega \cdot \omega
$$

If $c_{1}(E) \cdot \omega>0$, then we can assume $r_{c r}>1$.

Proof. Suppose $(A, 0)$ occurs as a reducible solution. Wedge $\omega$ with the perturbed equation,

$$
P_{+} F_{A}=t P_{+} F_{A_{0}}-i t \omega, \quad 0 \leq t \leq 1,
$$

then we have $c_{1}\left(K^{-1} \otimes E^{2}\right) \cdot \omega=t c_{1}\left(K^{-1}\right) \cdot \omega+t \omega \cdot \omega$. So

$$
(1-t) c_{1}\left(K^{-1} \otimes E^{2}\right) \cdot \omega=t c_{1}\left(E^{2}\right) \cdot \omega+t \omega \cdot \omega
$$

By the definition of $r_{c r}$, this gives (3.8).

If $c_{1}(E) \cdot \omega>0$, then $r_{c r}>0$. Notice that the right hand side of (3.8) is quadratic in $\omega$ while the left hand side is linear in $\omega$; by scaling $\omega$ by a positive constant, $r_{c r}$ can be chosen to be greater that 1 . The scaling would not affect the number of walls occurring in steps 2 and 3.

Corollary 2.3. If $\left(K^{-1}+2 E\right) \cdot \omega>0$ then there is an odd number of walls in steps 2 and 3 . If $\left(K^{-1}+2 E\right) \cdot \omega<0$ then there is an even number of walls in steps 2 and 3.

For symplectic manifolds with metrics of positive scalar curvature, first notice that such a manifold necessarily has $b_{2}^{+}=1$ by Taubes's Theorem 1 . If the wall crossing number for $K^{-1}$ is $n$, then since in Taubes's chamber, the SW invariant is \pm 1 , the invariant for the psc metric is $0=p n \pm 1$ for some integer $p$. But this is possible only if $n$ itself is \pm 1 . We have just proved the following: 
Lemma 2.4. If $M$ is a symplectic four-manifold with psc metric and $b_{2}^{+}=$ 1 , then the wall crossing number of $K^{-1}$, for a wall as described in steps 1-3 for any symplectic form, is \pm 1 .

This simple lemma put strong constraints on symplectic canonical class $K$ for symplectic manifolds with $b_{1} \neq 0$ if combined with the explicit wall crossing formula.

Lemma 2.5. Let $M$ be a symplectic four-manifold with psc metric and $b_{2}^{+}=1$. If $\omega_{p s c}$ is a harmonic self-dual two form associated to a positive scalar curvature metric and lies in the forward cone, then $K^{-1} \cdot \omega_{p s c}>0$.

Proof. By Lemma 2.4, the wall crossing number is nonzero, $K^{-1} \cdot \omega_{p s c}$ can not change sign. If $K^{-1} \cdot \omega_{p s c} \leq 0$, then since the space of metrics of positive scalar curvature is open, we can assume that $K^{-1} \cdot \omega_{p s c}<0$. Let $\omega$ be any symplectic form such that $K^{-1} \cdot \omega \neq 0$ (we can always choose such a symplectic form, since the space of symplectic forms is open in the space of closed forms). If $K^{-1} \cdot \omega>0$, there is an odd number of metric walls from any metric compatible with $\omega$ to any psc metric, and by Corollary 2.3 , there is an odd number of walls in step 2 and 3, so there is an even number of walls in step 1-3. If $K^{-1} \cdot \omega>0$, there is an even metric wall from any metric compatible with $\omega$ to any psc metric, and again by Corollary 2.3, there is an even number of walls in step 2 and 3, so again there is an even number of walls in step 1-3. But this contradicts with the fact that the $r \rightarrow \infty$ deformed SW equation has exactly one solution, since for positive scalar curvature metric, there is no solution for the standard SW equation.

Remark. This lemma can be proved directly for ruled surfaces by explicitly calculating $\omega_{p s c}$.

We give a simple but quite useful lemma which can be viewed as the analogue of Hodge index theorem of algebraic surfaces.

Lemma 2.6 (Light cone lemma). For smooth oriented four-manifold with $b_{2}^{+}=1$, any two elements $a, b$ in the closure of the forward cone have $a \cdot b \geq 0$. In particular, if $a, b$ are both nonzero, then $a \cdot b=0$ if and only if $b=\gamma a$ for some $\gamma>0$.

Proof. The quadratic form can be diagonalized in a real basis. Call the coordinate system $(x, \vec{y})$, then after a rescaling, it is represented as $x^{2}-$ $\sum_{i} y_{i}^{2}$. If $a=(x, \vec{y})$ and $b=\left(x^{\prime}, \vec{y}^{\prime}\right)$ are both in the closure of the forward cone, then

$$
x^{2}-\sum y_{i}^{2} \geq 0, \quad x^{\prime 2}-\sum y_{i}^{\prime 2} \geq 0, \quad x, x^{\prime} \geq 0
$$


Then Cauchy-Schwartz inequality implies $x x^{\prime} \geq \sqrt{\sum y_{i}^{2}} \sqrt{\sum{y_{i}^{\prime}}^{2}} \geq \sum y_{i} y_{i}^{\prime}$. So $a \cdot b \geq 0$.

If $a \cdot b \neq 0$, then $x \neq 0$ and $x^{\prime} \neq 0$. If $a \cdot b=0$, then the CauchySchwartz inequality becomes equality and $x^{2}=\sum y_{i}^{2}, x^{\prime 2}=\sum y_{i}^{\prime 2}$ and $\vec{y}$ is proportional to $\vec{y}$.

Finally, we prove a symplectic -1 curve theorem for symplectic manifolds with $b_{2}^{+}=1$. The idea of the proof comes from Taubes who cleverly uses reflections along -1 spheres in his proof of Theorem 6 . Recall for an oriented four-manifold $M$, the norm of a element $\xi \in H^{2}(M ; \mathbf{Z})$ is defined to be $N(\xi)=\frac{1}{2} \xi \cdot \xi$. For any $\eta \in H^{2}(M ; \mathbf{Z})$ with $\eta^{2}= \pm 1$ or \pm 2 , define a special kind of automorphism of $H^{2}(M ; \mathbf{Z})$, reflection $R(\eta)$ along $\eta$ by

$$
R(\eta): \xi \longrightarrow \xi-\frac{1}{N(\eta)}(\xi \cdot \eta) \eta
$$

Proof of Theorem A. Let us first assume that the wall crossing number for $K^{-1}$ is odd. The first step is to prove that $E$ is represented by a symplectic curve. In Taubes's chamber, the SW invariant of $K^{-1}$ is odd. By Corollary 2.3 , if $K^{-1} \cdot \omega>0$, the SW invariant of $K^{-1}$ for the metric compatible with $\omega$ is even, and if $K^{-1} \cdot \omega<0$, the SW invariant of $K^{-1}$ for the metric compatible with $\omega$ is odd.

Now apply Taubes's trick: Reflect along $E ; K^{-1}$ goes to $K^{-1}+2 E$. So, if $\left(K^{-1}+2 E\right) \cdot \operatorname{Re}(E)(\omega)>0(<0)$, then the SW invariant of $K^{-1}+2 E$ for the metric compatible with $\operatorname{Re}(E)(\omega)$ is even (odd). Note that $\operatorname{Re}(E)$ preserves the forward cone and the backward cone, so $\omega$ and $\operatorname{Re}(E)(\omega)$ are in the same cone. Apply Corollary 2.3; we see that no matter whether $\left(K^{-1}+2 E\right) \cdot \omega$ is positive or negative, the SW invariant of $K^{-1}+2 E$ is odd in Taubes's chamber, so $E$ is represented by a symplectic curve.

But it remains to prove that $E$ is indecomposable, i.e., $E$ can not be represented by disjoint union of symplectic curves. By the adjunction and dimension formulae, a generic connected, embedded, symplectic curve with negative square is a symplectic -1 curve. Suppose $F_{i}$ are components of the symplectic curve representing $E$. Then $\operatorname{dim}_{G r}\left(F_{i}\right)=0$, since $\operatorname{dim}_{G r}(E)=$ 0 . Since $E^{2}=-1$, at least one $F_{i}$ has negative square, say $F_{1}$, then $F_{1}$ is a symplectic -1 curve. Denote it by $E^{\prime}$. So $E$ is of the form $E^{\prime} \coprod_{i} F_{i}$. Let $T=\coprod_{i} F_{i}$. Since $E^{\prime}$ is a symplectic -1 curve, it must be unique, so the SW invariant of $K^{-1}+2 E^{\prime}$ is odd in Taubes's chamber. By Corollary 2.3 , if $\left(K^{-1}+2 E^{\prime}\right) \cdot \omega>0(<0)$, then the SW invariant of $K^{-1}+2 E^{\prime}$ for the metric compatible with $\omega$ is even (odd). Now apply Taubes's trick again: reflect along $E^{\prime}, K^{-1}+2 E^{\prime}$ goes to $K^{-1}-2 T$. Argued as above, 
we get that the SW invariant of $K^{-1}-2 T$ is odd in Taubes's chamber. So $-T$ is represented by a symplectic curve, but $T$ is also represented by a symplectic curve, a contradiction.

When the wall crossing number of $K^{-1}$ is even, then for $K^{-1}$-hence for $K^{-1}+2 E$ and $K^{-1}-2 T$ - the SW invariant is odd in any chamber, and the result follows from the same argument as above.

\section{Symplectic structures on ruled surfaces}

In this section we will prove Theorems B, C and D.

Theorem B. There is a unique symplectic structure on the $S^{2}$-bundle over a Riemann surface.

Let $M$ be a $S^{2}$-bundle, then $H^{2}(M ; \mathbf{Z})$ is a free abelian group of rank two generated by the base class $x$ and the fiber class $y$. Let $K_{s}^{-1}$ be the standard anticanonical class, then $K_{s}^{-1}=2 x+(2-2 g) y$ if $M$ is the trivial bundle, and $K_{s}^{-1}=2 x+(1-2 g) y$ if $M$ is the nontrivial bundle.

First we prove that for the standard canonical class, the symplectic structure is a deformation equivalent to the standard one. For the nontrivial $S^{2}$-bundle over $S^{2}$, we use Theorem A and Taubes's Theorem 5. For other $S^{2}$-bundles, we first show that the $r \longrightarrow \infty$ deformed SW equation has solutions for the fiber class $y$, then apply Taubes's argument of $S W=$ $\pm G r$ to conclude that $\operatorname{Gr}(y) \neq 0$. We finally prove that fiber class $y$ is indecomposable, i.e., can not be represented by disjoint union of embedded smooth submanifolds, so there exists $J$-holomorphic rational curve of selfintersection 0 in the fiber class. The last step is the following theorem of Mcduff:

Theorem. ([M]) Any minimal symplectic 4-manifold $(X, \omega)$ which contains an embedded rational curve $\Sigma$ with $\Sigma \cdot \Sigma \neq 0$ is deformation equivalent to the $S^{2}$-bundle over a Riemann surface with the standard symplectic form.

Then we show that exotic canonical classes, i.e., canonical classes which can not be mapped to the the standard ones by diffeomorphisms, do not support any symplectic forms.

Proof of Theorem B. We start from the chamber of psc metric, consider the SW equations for $K_{s}^{-1}+2 y . \quad S W\left(K_{s}^{-1}+2 y\right)=0$ for positive scalar curvature metric. Now consider the metric $g$ compatible with $\omega$. Do the deformation; when $r>>(a+b) / a b$ (where $[\omega]=a x+b y$ ), the SW invariant crosses an odd number of walls. By the wall crossing formula, the wall crossing number is odd for $K_{s}^{-1}+2 y$, hence there is at least one solution in Taubes's chamber. 
Lemma 3.1. Let $M$ be an $S^{2}$-bundle over $\Sigma$, then for any symplectic form $\omega$ whose canonical bundle is isomorphic to $K_{s}$, there is an odd number of walls crossed for $\operatorname{det}\left(S_{+}\right)=K_{s}^{-1}$ when going from the psc metric chamber to Taubes's chamber for the form $\omega$.

Proof. Since $y^{2}=0$, and $\omega_{p s c}$ is in the forward cone, $y \cdot \omega_{p s c}>0$, hence by Lemma 2.5, $\left(K_{s}^{-1}+2 y\right) \cdot \omega_{p s c}>0$. Let $\omega=a x+b y$ be any symplectic form. By a small deformation of $\omega$, we can assume that $\left(K_{s}^{-1}+2 y\right) \cdot \omega \neq 0$. If $\left(K_{s}^{-1}+2 y\right) \cdot \omega>0$, then by Corollary 2.3 , there is an odd number of walls in steps 2 and 3. Similarly, if $\left(K_{s}^{-1}+2 y\right) \cdot \omega<0$, by Corollary 2.3, there is an even number of walls in steps 2 and 3 . In both cases, odd numbers of walls occur.

Lemma 3.2. $y$ is an indecomposable class.

Proof. Let $y=(A x+B y) \coprod(C x+D y)$. If $M$ is the trivial $S^{2}$-bundle, then

$$
A D+B C=0, \quad A+C=0, \quad B+D=1 .
$$

Either $A=0$ or $1=2 B$. It is easy to see there is no nontrivial integer solution.

If $M$ is the nontrivial $S^{2}$-bundle, then

$$
A C+A D+B C=0, \quad A+C=0, \quad B+D=1 .
$$

We get $A(1-A-2 B)=0$. If $A=0$, then $C=0$. Since $B y$ and $C y$ are pseudo-holomorphic curves for the same symplecic form, $B$ and $C$ are all nonnegative, forcing one of them to be zero. If $A+2 B=1$, then $C+2 D=1$. The formal dimensions are

$$
\operatorname{dim}(A x+B y)=A(3-2 g)+1, \quad \operatorname{dim}(C x+D y)=C(3-2 g)+1 .
$$

Since the formal dimensions of both curves should be nonnegative, we see if $g \neq 1$, or $2, A$ and $C$ must both be zero, and $B=D=\frac{1}{2}$. But $y$ is a primitive class, so this is impossible. If $g=1$ or 2 , the only possible decomposition is $y=(y-x) \coprod x$. We will rule out this case by proving that $y-x$ is indecomposable and has too "small" a genus.

If $y-x=(A x+B y) \coprod(C x+D y)$, then we get $A(-B-A)+B(-1-A)=$ 0 . $B$ is divisible by $A$ provided $A \neq 0$. If $A=0$, then $C=-1, B=0$ and $D=-1$ : the trivial decomposition. So we assume $A \neq 0$. Write $B=k A$ to get $A=-k /(2 k+1)$. It has a nonzero integer solution only if $k=-1 \Rightarrow A=-1, B=1$ : the trivial decomposition again. So $y-x$ is indecomposable.

The genus of $y-x$ is 0 if $g=1$, and is -1 if $g=2$. 
The virtual genus of $y$ is zero; since $y$ is indecomposable, the $y$-curve must be an embedded symplectic sphere. If $M$ is not the nontrivial $S^{2}$ bundle over $S^{2}$, then $M$ does not have any - 1 sphere. By Mcduff's theorem, the symplectic structure for $K_{s}^{-1}$ is unique.

For the twisted $S^{2}$-bundle over $S^{2}$, the only anticanonical bundle is $2 x+y$, up to diffeomorphism. The class $x-y$ is represented by a -1 curve and its pairing with $K^{-1}$ is 1 , so by Theorem A, it is a symplectic -1 curve. Blow-down this curve to get a $C P^{2}$; by Taubes's Theorem 4, the symplectic structures on $C P^{2}$ are unique.

Next we want to show the other almost complex structures do not support any symplectic forms. We need the following result of $\mathrm{Wu}$.

Theorem. ([Wu]) Homotopy classes of almost complex structures on $M$ are in one-to-one correspondence with cohomology classes $K \in H^{2}(M ; \mathbf{Z})$ which are integral lifts of $w_{2}$ and satisfy $K^{2}=3 \sigma+2 \chi$.

The complex conjugation maps $x$ to $-x$ and $y$ to $-y$, so we can assume that the coefficient of $x$ is nonnegative. When the base is a sphere, it is easy to see that there are no exotic canonical classes. When the genus is one, if $M$ is the trivial bundle, the exotic canonical classes are $K=2 l x$, $l \geq 0$ or $K=2 l y, l \geq 0$; if $M$ is the nontrivial bundle, the exotic canonical classes are $K=(2 l+1) y, l>0$, or $K=l(2 x-y), l \geq 1$. When the genus is greater than 1 , if $M$ is the trivial bundle, the exotic canonical classes are $K=2 l x+\frac{4-4 g}{2 l} y, l \geq 1$ and $\frac{4-4 g}{2 l}$ is even; if $M$ is the nontrivial bundle, the exotic canonical classes are $K=2 l x+\left[\frac{2-2 g}{l}-l\right] y, \frac{2-2 g}{l}-l$ odd.

By Corollary 1, wall crossing numbers for these exotic canonical classes are not equal to \pm 1 ; by Lemma 2.4 , they do not support any symplectic structures.

We can also get complete constraints on the symplectic forms.

Proposition 3.3. Let $\omega=a x+$ by be a symplectic form on an $S^{2}$ fibre bundle over a Riemann surface $\Sigma$ whose symplectic canonical bundle has nonpositive coefficient on $x$. If the $S^{2}$-bundle is a product, then $a>0$, $b>0$; if the bundle is not, then $a>0, a+2 b>0$.

Proof. Suppose $a<0$. Let $e$ be the trivial class. Step 3 has no wall if $e$ is trivial. Step 2 has a wall if $K^{-1} \cdot \omega>0$ and does not have a wall if $K^{-1} \cdot \omega<$ 0 . The representative in the forward cone is $[-\omega]=(-a) x+(-b) y$. If step 2 has a wall, then $K^{-1} \cdot[-\omega]<0$, so there is a metric wall form $\omega_{p s c}$. If step 2 does not have any wall, then $K^{-1} \cdot[-\omega]>0$, so there are even numbers of metric walls. This implies in both cases that the deformed SW equation of $K^{-1}$ has even solutions, contradicting Taubes's theorem. 
Corollary 3.4. If $M$ is $C P^{2} \# \bar{C} P^{2}$, then $(x-y) \cdot \omega>0$; so the possible symplectic forms $\omega=a x+b y$ satisfy $a>0, b>0$.

Corollary 3.5. The symplectic cone of the standard canonical class $\mathcal{C}_{K_{s}}$ is the union of ample cones.

Proof. We only have to show symplectic cones are contained in some ample cones. For $S^{2}$ bundles with a base curve of genus $g$, there are Kahler ruled surface structures with $e \geq-g$. In Hartshorne's notation, $H^{2}(M ; \mathbf{Z})$ has basis $C_{0}$ and $F$ with $C_{0}^{2}=-e, C_{0} \cdot F=1$ and $F^{2}=0$. Let $D=\alpha C_{0}+\beta F$, then we have

Proposition 3.6. ([H] Cor 2.18, Prop 2.19 and 2.20) If $e \geq 0, D$ is ample if and only if $\alpha>0$ and $\beta>\alpha e$; if $e<0$, then $D$ is ample if and only if $\alpha>0$ and $\beta>\frac{1}{2} \alpha e$.

Our basis is related to Hartshorne's basis as follows: $y$ is just $F$, and

$$
x= \begin{cases}C+e / 2 F & \text { if } e \text { is even, } \\ C+(e+1) / 2 F & \text { if } e \text { is odd }\end{cases}
$$

It is easy to see for trivial bundles that the $e=0$ complex structure has the ample cone $a>0, b>0$, and for nontrivial bundles with base curve of genus greater that zero, the $e=-1$ complex structure has the ample cone $a>0, a+2 b>0$. For $C P^{2} \# \bar{C} P^{2}$, the $e=1$ complex structure has the ample cone $a>0, b>0$.

Now we prove Theorem C. Let $M_{k}$ be the blow-ups of the $S^{2}$-bundle with -1 curves $E_{1}, \cdots, E_{k}$. The anticanonical bundle of the standard almost complex structure $K^{-1}$ is $2 x+(2-2 g) y-\sum_{i} E_{i}$ for the trivial bundle and is $2 x+(1-2 g) y-\sum_{i} E_{i}$ for the nontrivial bundle. By Theorem A, for symplectic structures with the standard anticanonical bundle, $E_{i}$ are symplectic -1 curves, so we can symplectically blow-down $E_{i}$ and get back to $S^{2}$-bundles. Since the symplectic structures on $S^{2}$-bundles are unique, we also get

Proposition 3.7. The symplectic structures with the standard anticanonical bundle on $M_{k}$ are unique.

Let $K^{-1}=a x+b y+\sum_{i=1}^{k} c_{i} E_{i}$ be an anticanonical class on $M_{k}$. If $M$ is irrational, by Wall crossing formula and Lemma $2.4, a= \pm 2$ if $K^{-1}$ support some symplectic structures. Then Theorem will follow from Proposition 3.8 and the next Lemma. 
Lemma 3.8. Let $M$ be an irrational ruled surface, and $K^{-1}=2 x+b y+$ $\sum_{i=1}^{k} c_{i} E_{i}$ be an anticanonical class, then $K^{-1}$ is mapped to the standard one by some diffeomorphism.

Proof. A result of Friedman and Morgan $[\mathrm{FM}]$ is that an automorphism $\Psi$ of $H^{2}\left(M_{k} ; Z\right)$ comes from a diffeomorphism if and only if $\Psi y= \pm y$. It is easy to see that such automorphisms have the form

$$
\begin{gathered}
\Psi y= \pm y, \quad \Psi E_{i}=E_{i}+\alpha_{i} E_{i}, \quad i=1, \cdots, k, \\
\Psi x=x-\frac{\sum_{i=1}^{k} \alpha_{i}^{2}}{2} y+\sum_{i=1}^{k} \alpha_{i} E_{i}
\end{gathered}
$$

with $1 / 2 \sum_{i=1}^{k} \alpha_{i}^{2}$ being an integer. Again it is easy to see that any $K^{-1}=$ $2 x+b y+\sum_{i=1}^{k} c_{i} E_{i}$ is mapped to the standard $K^{-1}$ by such an automorphism.

The second statement of Theorem D follows from Proposition 3.7. To prove the first statement, we need to study the diffeomorphism group of $P_{k}^{2}$. Let $A_{u} t_{r}\left(P_{k}^{2}\right)$ be the group of automorphisms of $H^{2}(M ; \mathbf{Z})$ preserving the real characteristic classes $w_{2}$ and $p_{1}$. Since the manifolds are simply connected, an automorphism is just an automorphism of its quadratic form. The obvious automorphisms arise by multiplying some basis element by -1 and by permuting basis elements. Such automorphisms are called trivial. Reflections along classes with square -1 and -2 (for precise definition, see the paragraph before the proof of Theorem A) are also important as shown by the following theorem of Wall,

Theorem. [Wall]

(1) $\operatorname{Aut}_{r}\left(P_{2}^{2}\right)$ is generated by trivial automorphisms and $R\left(H-E_{1}-\right.$ $\left.E_{2}\right)$.

(2) Aut $_{r}\left(P_{k}^{2}\right)$ is generated by trivial automorphisms and $R\left(H-E_{1}-\right.$ $\left.E_{2}-E_{3}\right)$ when $3 \leq k \leq 9$.

(3) Every element of $A_{u} t_{r}\left(P_{k}^{2}\right)$ can be realized by a diffeomorphism.

Theorem D will be finished by the following proposition.

Proposition 3.9. Diffeomorphisms act transtively on the canonical classes of $P_{k}^{2}$ for $2 \leq k \leq 9$.

Proof. By Wall's theorem, it suffices to show that $A u t_{r}\left(P_{k}^{2}\right)$ acts transtively on all the characteristic elements $\xi$ with $\xi^{2}=9-k$. This is proved by Wall implicitly in $[\mathrm{W}]$ for $1 \leq k \leq 8$. We adapt his argument to prove the case $k=9$. Let $\xi=a H+\sum_{i=1}^{9} b_{i} E_{i}$ be a characteristic element with norm zero. 
Using trivial automorphisms, we can assume that $a, b_{i} \geq 0$ for $i=1, \cdots, 9$ and that the $b_{i}$ are arranged in decreasing order. Note

$$
\begin{aligned}
& R\left(H+E_{1}+E_{2}+E_{3}\right)\left(E_{i}\right)=E_{i}-\left(H+E_{1}+E_{2}+E_{3}\right), \quad i=1,2,3, \\
& R\left(H+E_{1}+E_{2}+E_{3}\right)\left(E_{i}\right)=E_{i}, \quad 4 \leq i \leq 9, \\
& R\left(H+E_{1}+E_{2}+E_{3}\right)(H)=2 H+E_{1}+E_{2}+E_{3} .
\end{aligned}
$$

This replaces $a$ by $2 a-b_{1}-b_{2}-b_{3}$. Hence we can decrease $a$ if $\mid 2 a-b_{2}-$ $b_{2}-b_{3} \mid<a$. We decrease $a$ until this is no longer satisfied. It is easy to see that it is impossible to have $3 a \leq b_{1}+b_{2}+b_{3}$, so $a \geq b_{1}+b_{2}+b_{3}$. Hence $0 \geq 2\left(b_{1} b_{2}+b_{1} b_{3}+b_{2} b_{3}\right) \geq \sum_{4 \leq i \leq 9} b_{i}^{2}$. Since the $b_{i}$ are in decreasing order, this implies that all the $b_{i}$ are the same. Since $\xi$ is the characteristic, and this is preserved by automorphisms in $\operatorname{Aut}_{r}\left(P_{k}^{2}\right)$, it is easy to see that $3 H+\sum_{1 \leq i \leq 9} E_{i}$ is the only class.

\section{Symplectic canonical classes and generalized adjunction inequality}

In this section, we prove Theorems $\mathrm{E}$ and $\mathrm{F}$.

Theorem E (Generalized adjunction inequality). Suppose $M$ is a symplectic four-manifold with $b_{2}^{+}=1$ and $\omega$ is the symplectic form. Let $C$ be a smooth connected embedded surface with nonnegative self-intersection. If $[C] \cdot \omega>0$, then $2 g(C)-2 \geq K \cdot[C]+[C]^{2}$.

As a corollary, we get the generalized Thom conjecture for symplectic four-manifolds with $b_{2}^{+}=1$.

Corollary 4.1. Suppose $M$ is a symplectic manifold with $b_{2}^{+}=1$ and $\omega$ is a symplectic form in the forward cone. Let $C$ be a smooth, connected embedded surface with nonnegative self intersection. If the class $[C]$ is also represented by a connected, embedded symplectic surface $\Sigma$, then $g(C) \geq$ $g(\Sigma)$.

The following theorem of Kronheimer and Mrowka is crucial in our proof.

Theorem. ([KM]) If the $S W$ equation of $\mathcal{L}$ on a cylinder $\Sigma \times S^{1} \times R$ has translation invariant solutions, then if $g(\Sigma) \geq 1$,

$$
\left|c_{1}(\mathcal{L}) \cdot[\Sigma]\right| \leq 2 g-2 .
$$

Proof of Theorem E. First blow-up symplectically, until $[C] \cdot[C]=0$. It is important that we still have $[C] \cdot \omega>0$, but $[C]$ is in the boundary of the forward cone. 
If $K \cdot[C]<0$, then $K \cdot[C] \leq-2$ since $K$ is characteristic and the adjunction inequality is trivially true.

If $K \cdot[C]=0$ only when $C$ is a sphere, the adjunction inequality does not hold. Blow-up symplectically once more, then $C-E$ is represented by a sphere with square -1 and has pairing 1 with $K^{\prime}=K+E$. By Theorem A, $E-C$ can be represented by a symplectic -1 curve $C^{\prime}$. Note that $[E]=\left[C^{\prime}\right]+[C]$ and $[C]^{2}=0$; apply a reflection along $E$ : $K^{-1 \prime}$ goes to $K^{-1^{\prime}}-2[C]$. Argued as in the proof of Theorem $\mathrm{A},-[C]$ is represented by a symplectic curve, but $[C] \cdot \omega>0$, a contradiction.

The remaining case is $K \cdot[C]>0$. If the wall crossing number of $K^{-1}$ is even, one can directly generate a SW solution for the long neck metric. Let $\omega_{L N}$ be the unique self-dual harmonic form for the long neck metric ( $\omega_{L N}$ may not be symplectic). If the wall crossing number of $K^{-1}$ is odd, assume first that $K^{-1} \cdot \omega_{L N}<0$ where now the neck is taken with respect to the smooth surface $C$. Now $K^{-1} \cdot[C]<0$. Start from Taubes's chamber; the invariant of $K^{-1}$ is \pm 1 . If $K^{-1} \cdot \omega>0$, then in the symplectic metric chamber, the invariant is 0 modulo 2 by Corollary 2.3 ; if $K^{-1} \cdot \omega<0$, in the symplectic metric chamber, the invariant is 1 modulo 2 by Corollary 2.3. In either case, the invariant is 1 modulo 2 in the long neck chamber, so a solution exists there. If $C$ is a sphere, then the cylinder has psc metric, but this implies that there is no solution. Therefore, we can assume that $g(C) \geq 1$. By KM's argument, there exists a time-independent solution of SW for $K^{-1}$, so $2 g(C)-2 \geq K \cdot[C]$ by KM's theorem.

It remains to prove $K^{-1} \cdot \omega_{L N}<0$, or equivalently, $K \cdot \omega_{L N}>0$; we need the light cone lemma. By stretching the neck to infinite length and normalizing $\omega_{L N}$ by $\int_{M} \omega_{L N}^{2}=1$, we get $\omega_{L N} \cdot[C]$ goes to zero, as argued by Kronheimer and Mrowka. Since the the closure of the forward cone is closed but noncompact, $\omega_{L N}$ can either converge to some finite point $\omega_{L N}^{\infty}$ with $\int_{M} \omega_{L N}^{\infty^{2}}=1$, or goes to infinity along the hyperbola. The first case can not happen. Since $\int_{M} \omega_{L N}^{\infty^{2}}=1, \omega_{L N}^{\infty}$ is in the interior of the forward cone. So $\omega_{L N}$ can not stay inside finite regions of the forward cone because $\omega_{L N}^{\infty} \cdot[C]=0$ and $[C] \cdot[C]=0$ (light cone lemma again). Therefore, written in terms of the basis in the light cone lemma, the $x$-coordinate of $\omega_{L N}$ also goes to infinity. Let us do another normalization by fixing its $x$-coordinate to be 1. Under this new normalization, $\omega_{L N} \cdot[C]$ still goes to zero. Now $\omega_{L N}$ must converge to a finite point, since the set of points with $x$-coordinate equal to 1 is compact. Still denote the accumulation point by $\omega_{L N}^{\infty}$. Since $\omega_{L N}^{\infty} \cdot[C]=0$ and $[C] \cdot[C]=0$, by the light cone lemma, $\omega_{L N}^{\infty}=\gamma[C]$ for some $\gamma>0$. So $K \cdot \omega_{L N}^{\infty}=\gamma(K \cdot[C])>0$. But this implies that $K \cdot \omega_{L N}>0$ if we choose a long enough neck. 
Remark. We largely follow KM's proof of the Thom conjecture for $C P^{2}$. Our key observation is that it is sometimes better to work in the symplectic category than in the Kahler category with the powerful results of Taubes and the general wall crossing formula.

Remark. Let $M, \omega$ be as in Theorem E. For an immersed surface $C$ with $q$ positive intersection points and no negative intersection points such that $[C] \cdot \omega>0$ and $[C]^{2}-2 q \geq 0$, we still can prove $2 g(C)-2 \geq K \cdot[C]+[C]^{2}-2 q$.

Theorem F. For a symplectic four-manifold with $b_{2}^{+}=1$, let $\omega_{1}$ and $\omega_{2}$ be two symplectic forms with $\omega_{1}$ in the forward cone. Let $K_{1}$ and $K_{2}$ be the two symplectic canonical classes for $\omega_{1}$ and $\omega_{2}$. If $\omega_{1} \cdot \omega_{2}>0$, then $K_{1} \cdot \omega_{1} \geq K_{2} \cdot \omega_{1}$, where equality holds if and only if $K_{1}=K_{2}$ modulo torsion. If $\omega_{1} \cdot \omega_{2}<0$, then $K_{1} \cdot \omega_{1} \geq-K_{2} \cdot \omega_{1}$.

To prove the Theorem, we need the following important theorem of Donaldson.

Theorem. (Donaldson [D]) Let $M$ be a four-manifold with $\omega$ an integral symplectic form. Then for $k$ large, there is an irreducible embedded symplectic curve $\Sigma$ dual to $k \omega$.

Proof of Theorem $F$. After perturbing $\omega_{1}$ if necessary, we can assume that $\omega \in H^{2}(M ; Q)$. Multiply $\omega$ by some integer $N$; we can further assume $\omega_{1} \in$ $H^{2}(M ; Z)$. Apply Donaldson's theorem; we get an irreducible embedded curve $\Sigma$ dual to $k \omega_{1}$. Take $k$ large enough, then $K^{1} \cdot[\Sigma]+[\Sigma]^{2}>0$ and $K^{2} \cdot[\Sigma]+[\Sigma]^{2}>0$, so $\Sigma$ is not a sphere. To apply Theorem $\mathrm{E}$ to $\Sigma, K^{2}$ and $\omega_{2}$, we only have to check that $\omega_{2} \cdot[\Sigma]>0$, but this follows from the fact that $\omega_{1} \cdot \omega_{2}>0$. By Theorem E, we get $K_{1} \cdot[\Sigma]+[\Sigma]^{2}=2 g(\Sigma)-2 \geq K_{2} \cdot[\Sigma]+[\Sigma]^{2}$. Therefore, $K_{1} \cdot[\Sigma] \geq K_{2} \cdot[\Sigma]$, but $[\Sigma]=k \omega_{1}$. Hence,

$$
K_{1} \cdot \omega_{1} \geq K_{2} \cdot \omega_{1} .
$$

Since rational symplectic forms are dense, we have $K_{1} \cdot \omega_{1} \geq K_{2} \cdot \omega_{1}$ for all possible $\omega_{1}$. If $K_{1}-K_{2}$ is not torsion, the equality holds for some symplectic form $\omega_{0}$. Then $K_{1}-K_{2}$ can take both signs for symplectic forms near $\omega_{0}$, violating the above inequality.

\section{Remark.}

(1) Taubes's original argument can go through directly under the assumption that either $M$ has psc metric or the wall crossing number for $K_{2}^{-1}$ is even.

(2) The above argument is very general; in the case of $b_{2}^{+}>1$, by replacing $K_{2}^{-1}$ with a basic SW class, one can give another proof of Taubes's more constraint on basic classes [T2]. 
Corollary 4.3. If $K_{1}$ and $K_{2}$ are two distinct symplectic canonical classes, then the symplectic cones do not intersect.

Corollary 4.4. If $f$ is a diffeomorphism such that $f^{*} K_{1} \neq K_{1}$ modulo torsion, then the symplectic cone does not intersect with its image under $f^{*}$.

This gives constraints on the symplectic cones and diffeomorphisms.

\section{Acknowledgement}

The authors are deeply grateful to Prof. Yau for introducing us into this field and his constant encouragement. The authors thank Prof. Taubes for carefully reading this paper and pointing out numerous mistakes, and above all, for sharing his important ideas with us.

\section{References}

[B] R. Brussee, Some $C^{\infty}$-properties of Kahler surfaces, preprint.

[BT] R. Bott and L. Tu, Differential forms in algebraic topology, Springer, 1982.

[D] S. K. Donaldson, talks given at M.I.T.

[DK] S. K. Donaldson and P. Kronheimer, The geometry of four-manifolds, Clarendon Press, Oxford, 1990.

[FKMST] R. Fintushel, P. Kronheimer, T. Mrowka, R. Stern and C. H. Taubes, (to appear).

[FM1] R. Friedman and J. Morgan, Algebraic surfaces and Seiberg-Witten invariants, preprint.

[FM2] _ Smooth four-manifolds and complex surfaces, Springer, 1994.

[G] M. Gromov, Pseudo holomorphic curves in symplectic manifolds, Invent. Math. 82 (1985), 307-347.

[KM] P. Kronheimer and T. Mrowka, The genus of embedded surfaces in the projective plane, Math. Res. Letters 1 (1994), 797-808.

[LL] T. J. Li and A. Liu, General wall crossing formula, preprint.

[M] D. Mcduff, The structure of rational and ruled symplectic 4-manifold, J. AMS 1 (1990), 679-710.

[MST] J. Morgan, Z. Szabo and C. H. Taubes, The generalized Thom conjecture, in preparation.

[R] Y. Ruan, Topological sigma model and Donaldson type invariants in Gromov theory, preprint.

[RT] Y. Ruan and G. Tian, A mathematical theory of quantum cohomology, preprint.

[SW] N. Seiberg and E. Witten, Electric-magnetic duality, monople condensation, and confinement in $N=2$ supersymmetric Yang-Mills theory, Nuclear Physics B 426 (1994), 19-52.

[T1] C. H. Taubes, The Seiberg-Witten invariants and symplectic forms, Math. Res. Letters 1 (1994), 809-822.

[T2] More constraints on symplectic manifolds from Seiberg-Witten equations, Math. Res. Letters 2 (1995), 9-14.

[T3] _ The Seiberg-Witten invariants and Gromov invariants, preprint. 
[T4] talks given at Harvard.

[Wa] C. T. C. Wall, On the orthogonal groups of unimodular quadratic forms, II, J. Reine and Angew. Math. 213 (1963), 323-338.

[Wi] E. Witten, Monoples and 4-manifolds, Math. Res. Letters 1 (1994), 769-796.

[Wu] W. T. Wu, Sur les classes caractéristiques des structures fibrées sphériques, Actualités et industrielles, Hermann, Paris, 1952.

[Y] S. T. Yau, Calabi's conjecture and some new results in algebraic geometry, Proc. Nat. Acad. Sci. 74 (1977), 1789-1799.

Department of Mathematics, Brandeis Univeritiy, Waltham M A 02254

E-mail address: tli@math.harvard.edu

Department of Mathematics, Harvard University, Cambridge, M A 02138

E-mail address: akliu@math.harvard.edu 